\title{
A study of the chromospherically active binaries UX Fornacis and AG Doradus*
}

\author{
A. Washuettl ${ }^{1,2}$ and K. G. Strassmeier ${ }^{1,2}$ \\ 1 Astrophysical Institute Potsdam (AIP), An der Sternwarte 16, 14482 Potsdam, Germany \\ 2 Institute for Astronomy, University of Vienna, Türkenschanzstraße 17, 1180 Wien, Austria \\ e-mail: AWashuettl / KStrassmeier@aip.de
}

Received 21 September 2000 / Accepted 29 January 2001

\begin{abstract}
This paper presents a time-series spectrum analysis of the two chromospherically active binary stars, UX Fornacis and AG Doradus. To investigate the rotational behaviour, in combination with activity in the $\mathrm{H} \alpha$ line, we obtained almost 40 high-resolution, high- $S / N$ spectra for each system. Both binaries, UX For and AG Dor, show $\mathrm{H} \alpha$ of the primary star in absorption but filled in by chromospheric emission while the $\mathrm{H} \alpha$ line of the secondaries appear in pure emission. Line variations are seen and are approximately 5- $\sigma$ detections but are not linked to the orbital nor the rotational periods and seem to be erratic. We obtained new orbital elements for both systems and used them in combination with Hipparcos parallaxes and our $\mathrm{H} \alpha$-spectrum synthesis to determine absolute parameters. We present the first Doppler image of the AG Dor primary using line-profile variations of the Fe I $\lambda 6546$ line. The image reveals a cool polar feature and three equatorial spots with an average surface temperature difference, photosphere minus spots, of up to $1500 \mathrm{~K}$ for the polar spot and $\approx 800 \mathrm{~K}$ for the equatorial spots. Since AG Dor is one of the slowest rotators $\left(v \sin i=18 \mathrm{~km} \mathrm{~s}^{-1}\right)$ that has ever been Doppler imaged, we present several tests to demonstrate the external consistency of our map.
\end{abstract}

Key words. stars: activity - stars: atmospheres - stars: imaging - stars: AG Dor, UX For - stars: late-type binaries: close

\section{Introduction}

The generally accepted explanation for photospheric variations of chromospherically active binary stars is that their surfaces are covered with cool starspots. There are only very few stars that are chromospherically active and have no photospheric rotational variability due to starspots (e.g. the Hertzsprung-gap giants 31 Comae and $\psi^{3}$ Psc; Bopp et al. 1988; Strassmeier et al. 1994). The vast majority, however, shows chromospheric and photospheric activity (along with coronal activity if measured) that suggest an organised magnetic field as the mutual cause because in-phase rotational modulation is usually detected at all three atmospheric levels. Just to name a few examples, Rodonò et al. (1987) presented evidence of a close spatial correlation between spots and plage-like features on the RS CVn binary II Peg, as on the Sun, while Catalano et al. (1996) found a systematic lag of $30^{\circ}-50^{\circ}$ between plages and spots on a set of five very active RS CVn binaries. Doyle et al. (1989) found evidence for a rotational modulation in chromospheric as well as transition-region lines. Our disk resolved Doppler-imaging

Send offprint requests to: A. Washuettl,

e-mail: awashuettl@aip.de

* Based on observations obtained at the European Southern Observatory, La Silla, Chile. data of RS CVn binaries confirm the spatial relation of spots with CaII $\mathrm{H}$ and $\mathrm{K}$ emission regions as well as with $\mathrm{H} \alpha$ emission and absorption (e.g. for the K0III-IV binary HU Vir; Strassmeier 1994; Hatzes 1998). Furthermore, Collier Cameron \& Robinson (1989; and subsequent papers) observed transient absorption features in $\mathrm{H} \alpha$ due to prominences corotating with the star.

A large piece of information about the solar chromosphere comes from observations of the Balmer $\mathrm{H} \alpha$ line. Analogous to the Sun, we expect that stellar plages are seen in the $\mathrm{H} \alpha$ line as emission components while prominences show up as transient absorption features. $\mathrm{H} \alpha$ contains information about the photosphere, the chromosphere, and about activity tracers such as plages, prominences and macroscopic velocity fields (see e.g. Oliveira \& Foing 1999). $\mathrm{H} \alpha$ is easy to observe thanks to efficient detectors and spectrographs in the red part of the spectrum. Among the class of chromospherically active binaries, only the most active systems show $\mathrm{H} \alpha$ as a pure emission line. Commonly, it is seen in absorption partly filled in by chromospheric emission. The amount of filling-in often varies and is used to detect rotational modulation from the stellar chromosphere, preferably for red dwarf stars that become relatively faint in the Ca II $\mathrm{H}$ and $\mathrm{K}$ region (e.g. Giampapa et al. 1989). 
For the present study, we chose two binary systems with rapidly-rotating $\mathrm{G}-\mathrm{K}$-dwarf components. Both systems, UX For $(\mathrm{G} 6+\mathrm{K} 0)$ and $\mathrm{AG}$ Dor $(\mathrm{K} 0+\mathrm{K} 4)$, show $\mathrm{H} \alpha$ from the primary star as an absorption line filled in by chromospheric emission while the $\mathrm{H} \alpha$-line of the secondary star is in pure emission. The first system, UX For $=$ HD 17084, shows strong Ca II H and K emission lines, moderate $\mathrm{Li}$ abundance and strong 6 - $\mathrm{cm}$ radio emission (Lloyd-Evans \& Koen 1987; Henry et al. 1996; Randich et al. 1993; Strassmeier et al. 1993). It was also detected by the ROSAT and EUVE satellites (Pye et al. 1995; Bowyer et al. 1996). Bidelman \& MacConnell (1973) determined a spectral type of G5 IV while Houk (1982) listed G5-8 V + (G). Lloyd-Evans \& Koen (1987) found a photometric period of 0.957 days. The light-curve amplitude as well as the mean brightness are variable. No colour variation was detected. Based on the combined colours, Cutispoto (1998) derived a spectral classification of $\mathrm{G} 6 \mathrm{~V}+\mathrm{K} 3 \mathrm{~V}$.

The second system, AG Dor $=$ HD 26354, shows only weak CaII $\mathrm{H}$ and $\mathrm{K}$ emission lines (Bidelman \& MacConnell 1973; Houk \& Cowley 1975) and a low lithium abundance of less than solar (Pallavicini et al. 1992). However, it was detected by the EUVE satellite (Bowyer et al. 1996). Lloyd-Evans \& Koen (1987) reported light modulations with a period of 2.533 days and an amplitude of 0.05 in $V$, in agreement with the orbital period. Cutispoto (1998) found a $V$-amplitude of about 0.04 in February 1992, the smallest so far observed, and only very weak colour variations. At this time, AG Dor was brighter than in any previous epoch, confirming the presence of long-term variability in the global spottedness (Cutispoto 1998). Amado et al. (1999) determined spot temperatures by making use of the light-curve spot-modelling technique and the molecular TiO bandhead strength. They found a photospheric temperature of $5000 \mathrm{~K}$ and spot temperatures of 4000-4600K. Houk (1978) determined the spectral type as K1 Vp. A refined broad-band colourdisentangling technique along with accurately measured combined colours led Cutispoto to a classification of K1$2 \mathrm{~V}+\mathrm{K} 6 \mathrm{~V}$ (Cutispoto 1992) and K1 V + K5 V (Cutispoto 1998), in good agreement with the spectroscopically determined classification from Houk (1978). However, Balona (1987) found no evidence for a K5-6V secondary component in his radial-velocity spectra. Such a secondary would be expected to be $\Delta V \approx 1^{\mathrm{m}} 5$ fainter than a K1-2 primary and should be visible in AG Dor's spectra.

Our study aims to detect a correlation between activity in the $\mathrm{H} \alpha$-chromosphere and activity in the stellar photosphere. First, we determine the absolute parameters and present new double-lined orbits for both systems. Then, we disentangle the two spectra for each of the binary systems and extract $\mathrm{H} \alpha$ equivalent widths and photospheric absorption line profiles. While the former are used to construct dynamic chromospheric spectra, the latter are used - at least for AG Dor - to obtain its first photospheric Doppler image. For UX For, $S / N$ turned out to be too low for Doppler imaging.

\section{Observations and data reduction}

All observations were made with the ESO coudé auxiliary telescope (CAT) and the coudé echelle spectrograph (CES) during the period November 18-24, 1992. We used a $1024 \times 1024$ pixel CCD at a resolving power of 50000 (spectral resolution of $\approx 0.12 \AA$ ). The integration time of the spectra was between 1200 and 3600 s, which allowed for an average signal-to-noise ratio of 70:1 for UX For and 150:1 for AG Dor. Each spectrum covers $\approx 50 \AA$ from $\lambda 6526 \AA$ to $\lambda 6576 \AA$. A typical night of observations includes two biases, 10 flat-field images, $5-7$ thorium-argon lamp integrations at the beginning and at the end of each night, and 1-3 integrations of a rapidly rotating B-star (for telluric line correction).

All data were reduced in the same standard fashion using the Image Reduction and Analysis Facility (IRAF) and included bias subtraction, flat fielding, optimal aperture extraction, and telluric line division. The flat-field exposures were averaged together and used to remove the pixel-to-pixel variation of the detector sensitivity. Th-Ar comparison spectra and spectra of a bright radial-velocity standard star were obtained at least once during each night to ensure an accurate wavelength calibration.

A total of 37 spectra of UX For (see Table 1) and 38 spectra of AG Dor (Table 2) were obtained. Additionally, a total of 14 bright-spectrum comparison stars were observed throughout the run. We chose stars with well established spectral classifications (if possible MK standards) and with spectral lines not significantly broadened by rotation.

$\mathrm{H} \alpha$ spectra are usually contaminated with many small absorption lines due to water vapor in the terrestrial atmosphere. Spectra of rapidly rotating hot stars show mostly a flat continuum near $\mathrm{H} \alpha$ and are ideally suited to reconstruct the nightly telluric spectrum. We chose the rapidly rotating B star HR 674 (spectral type: B8 V$\mathrm{IV}, v \sin i \approx 250 \mathrm{~km} \mathrm{~s}^{-1}$ ). Several nightly spectra were combined to increase the signal-to-noise ratio and eliminate cosmic-ray hits. High-frequency noise was eliminated by setting all intensity values of the normalized B-star spectrum above 0.985 to unity. The remaining spectra, weighted by airmass, were then used for dividing the object spectra.

\section{Astrophysical parameters of UX For and AG Dor}

\subsection{Radial velocities and orbit}

Radial velocities for AG Dor were determined from crosscorrelations with spectra of the radial velocity standard star HR $1829\left(v_{\mathrm{r}}=-13.5 \mathrm{~km} \mathrm{~s}^{-1}\right.$; Blumberg \& Boksenberg 2000), observed during the same night. The cross correlations were computed using IRAF's fxcor routine (see Fitzpatrick 1993), which fits a Gaussian to the cross-correlation function to determine the central shift. Because the $\mathrm{H} \alpha$ line can be severely affected by velocity 
Table 1. Log of the observations of UX Fornacis and radial velocities

\begin{tabular}{|c|c|c|c|c|c|}
\hline $\begin{array}{r}\text { UT Date } \\
(1992)\end{array}$ & $\begin{array}{c}\text { HJD } \\
(24489+)\end{array}$ & phase & $\begin{array}{c}v_{\mathrm{r}}^{\mathrm{P}} \\
{\left[\mathrm{km} \mathrm{s}^{-1}\right]}\end{array}$ & $\begin{array}{c}v_{\mathrm{r}}^{\mathrm{S}} \\
{\left[\mathrm{km} \mathrm{s}^{-1}\right]}\end{array}$ & $\begin{array}{l}\Delta t \\
{[\mathrm{~s}]}\end{array}$ \\
\hline Nov 19 & 45.5423 & 0.3221 & -20.3 & 89.6 & 3600 \\
\hline 19 & 45.7263 & 0.5148 & -76.9 & 151.9 & 1800 \\
\hline 19 & 45.7492 & 0.5387 & -73.9 & 147.5 & 1800 \\
\hline 19 & 45.7694 & 0.5599 & -70.1 & 142.8 & 1200 \\
\hline 19 & 45.8388 & 0.6326 & -45.7 & 104.5 & 1200 \\
\hline 19 & 45.8532 & 0.6477 & -40.3 & 99.4 & 1050 \\
\hline 20 & 46.5061 & 0.3315 & -29.2 & 85.7 & 1200 \\
\hline 20 & 46.5249 & 0.3512 & -39.7 & 95.3 & 1200 \\
\hline 20 & 46.5401 & 0.3671 & -44.8 & 109.1 & 1200 \\
\hline 20 & 46.5547 & 0.3824 & -51.3 & 114.2 & 1200 \\
\hline 20 & 46.5700 & 0.3984 & -58.1 & 123.8 & 1200 \\
\hline 20 & 46.5846 & 0.4137 & -62.3 & 133.7 & 1200 \\
\hline 20 & 46.5998 & 0.4296 & -67.9 & 140.8 & 1200 \\
\hline 20 & 46.6151 & 0.4456 & -70.9 & 143.9 & 1200 \\
\hline 20 & 46.6582 & 0.4908 & -77.0 & 151.7 & 1200 \\
\hline 20 & 46.7269 & 0.5627 & -72.7 & 140.9 & 1200 \\
\hline 20 & 46.7422 & 0.5787 & -66.1 & 140.0 & 1200 \\
\hline 20 & 46.7575 & 0.5948 & -61.7 & 134.9 & 1200 \\
\hline 20 & 46.7721 & 0.6101 & -56.7 & 127.3 & 1200 \\
\hline 20 & 46.7873 & 0.6260 & -51.0 & 106.1 & 1200 \\
\hline 20 & 46.8026 & 0.6420 & -44.1 & 98.2 & 1200 \\
\hline 20 & 46.8172 & 0.6573 & -33.8 & 94.2 & 1200 \\
\hline 20 & 46.8325 & 0.6733 & -27.4 & 88.9 & 1200 \\
\hline 20 & 46.8477 & 0.6892 & -18.8 & 82.9 & 1200 \\
\hline 21 & 47.5368 & 0.4109 & -60.4 & 129.0 & 1200 \\
\hline 21 & 47.5519 & 0.4268 & -65.8 & 135.0 & 1200 \\
\hline 21 & 47.8242 & 0.7119 & - & - & 1080 \\
\hline 21 & 47.8377 & 0.7261 & - & - & 1140 \\
\hline 21 & 47.8493 & 0.7382 & - & - & 720 \\
\hline 22 & 48.5365 & 0.4580 & -71.4 & 141.6 & 1200 \\
\hline 22 & 48.5525 & 0.4747 & -71.6 & 143.8 & 1200 \\
\hline 23 & 49.7678 & 0.7475 & - & - & 1500 \\
\hline 23 & 49.7862 & 0.7668 & - & - & 1500 \\
\hline 23 & 49.8042 & 0.7857 & - & - & 1320 \\
\hline 24 & 50.7710 & 0.7982 & - & - & 1200 \\
\hline 24 & 50.7873 & 0.8153 & 64.2 & -45.1 & 1500 \\
\hline 24 & 50.8061 & 0.8350 & 73.4 & -44.0 & 1500 \\
\hline
\end{tabular}

fields originating from microflares or a stellar wind, the $\mathrm{H} \alpha$ region was excluded from the cross correlations. However, the secondary of AG Dor shows up only as a weak emission line in $\mathrm{H} \alpha$. Its radial velocities were measured from the residual spectra after subtraction of a reference spectrum (Sect. 3.2).

In case of UX For, Fe I $\lambda 6546 \AA$ is the only line that significantly contributes to the cross-correlation function besides the $\mathrm{H} \alpha$ line. Consequently, the radial velocity of $\lambda 6546$ was measured directly by fitting a double Gaussian to the primary and secondary line using IRAF's splot routine. In seven out of 37 cases, the primary and secondary lines were severely blended and no reliable radial velocities could be obtained. All other observations were given equal weight when calculating the orbit.
Table 2. Observing log of AG Doradus and radial velocities

\begin{tabular}{|c|c|c|c|c|c|}
\hline $\begin{array}{r}\text { UT Date } \\
(1992)\end{array}$ & $\begin{array}{c}\text { HJD } \\
(24489+)\end{array}$ & phase & $\begin{array}{c}v_{\mathrm{r}}^{\mathrm{P}} \\
{\left[\mathrm{km} \mathrm{s}^{-1}\right]}\end{array}$ & $\begin{array}{c}v_{\mathrm{r}}^{\mathrm{S}, \mathrm{H}_{\alpha} \mathrm{res}} \\
{\left[\mathrm{km} \mathrm{s}^{-1}\right]}\end{array}$ & $\begin{array}{l}\Delta t \\
{[\mathrm{~s}]}\end{array}$ \\
\hline Nov 19 & 45.5867 & 0.0354 & 124.5 & -26.9 & 3600 \\
\hline 19 & 45.7944 & 0.1165 & 111.8 & -4.9 & 1800 \\
\hline 19 & 45.8173 & 0.1254 & 110.1 & -2.4 & 1800 \\
\hline 20 & 46.6783 & 0.4615 & 14.9 & 171.6 & 1800 \\
\hline 20 & 46.6998 & 0.4699 & 14.1 & 171.7 & 1800 \\
\hline 21 & 47.5712 & 0.8100 & 86.0 & 37.1 & 1800 \\
\hline 21 & 47.5933 & 0.8186 & 88.9 & 20.3 & 1800 \\
\hline 21 & 47.6413 & 0.8374 & 95.1 & 23.4 & 1800 \\
\hline 21 & 47.6633 & 0.8459 & 98.3 & 22.5 & 1800 \\
\hline 21 & 47.7161 & 0.8665 & 104.6 & 15.3 & 1800 \\
\hline 21 & 47.7381 & 0.8751 & 107.1 & 11.3 & 1800 \\
\hline 21 & 47.7818 & 0.8922 & 111.5 & 5.6 & 1800 \\
\hline 21 & 47.8037 & 0.9007 & 113.8 & 3.3 & 1800 \\
\hline 22 & 48.5740 & 0.2014 & 87.7 & 20.1 & 1800 \\
\hline 22 & 48.5955 & 0.2098 & 84.8 & 20.4 & 1800 \\
\hline 22 & 48.6337 & 0.2247 & 79.9 & 26.0 & 1800 \\
\hline 22 & 48.6552 & 0.2331 & 77.3 & 37.2 & 1800 \\
\hline 22 & 48.6774 & 0.2418 & 74.1 & 48.1 & 1800 \\
\hline 22 & 48.6990 & 0.2502 & 71.2 & $(7.4)$ & 1800 \\
\hline 22 & 48.7337 & 0.2637 & 66.6 & 55.2 & 1800 \\
\hline 22 & 48.7559 & 0.2724 & 63.6 & 63.9 & 1800 \\
\hline 22 & 48.7788 & 0.2813 & 60.4 & $(34.5)$ & 1800 \\
\hline 22 & 48.8274 & 0.3003 & 54.1 & $(44.7)$ & 1800 \\
\hline 22 & 48.8497 & 0.3090 & 51.5 & $(31.8)$ & 1800 \\
\hline 23 & 49.5635 & 0.5876 & 20.4 & 163.1 & 1800 \\
\hline 23 & 49.5857 & 0.5963 & 21.6 & 159.3 & 1800 \\
\hline 23 & 49.6278 & 0.6127 & 24.8 & 149.7 & 2400 \\
\hline 23 & 49.6567 & 0.6240 & 26.5 & 148.2 & 2400 \\
\hline 23 & 49.6961 & 0.6394 & 31.3 & 139.6 & 2100 \\
\hline 23 & 49.7216 & 0.6493 & 33.6 & 134.3 & 2100 \\
\hline 23 & 49.7456 & 0.6587 & 36.1 & 134.3 & 1800 \\
\hline 23 & 49.8257 & 0.6900 & 45.9 & 129.8 & 2100 \\
\hline 23 & 49.8512 & 0.6999 & 48.9 & 109.3 & 2100 \\
\hline 24 & 50.5627 & 0.9776 & 124.7 & -20.7 & 3600 \\
\hline 24 & 50.6190 & 0.9996 & 125.5 & -24.2 & 3600 \\
\hline 24 & 50.6655 & 0.0178 & 125.5 & -22.7 & 3600 \\
\hline 24 & 50.7415 & 0.0474 & 123.9 & -21.4 & 3600 \\
\hline 24 & 50.8384 & 0.0852 & 118.7 & -14.2 & 3480 \\
\hline
\end{tabular}

Orbital elements were derived with the differentialcorrection program of Barker et al. (1967), as modified and described by Fekel et al. (1999). Our final elements for both binaries converged at an eccentricity so close to zero that a formal zero-eccentricity solution was adopted (see Lucy \& Sweeney 1971). The standard error of an observation of unit weight is $4.62 \mathrm{~km} \mathrm{~s}^{-1}$ for UX For, using all available measurements of the primary and secondary component. Some of Balona's (1987) O-C residuals are $12 \mathrm{~km} \mathrm{~s}^{-1}$, and two of Balona's secondary $\mathrm{O}-\mathrm{C}$ residuals are as large as $23 \mathrm{~km} \mathrm{~s}^{-1}$. The standard error for the AG Dor primary was $0.41 \mathrm{kms}^{-1}$, and was calculated using all 38 cross-correlation measurements. The secondarystar orbit was computed independently from the primary star by using the radial velocities of the residual $\mathrm{H} \alpha$ emission feature (see Sect. 4). $\mathrm{H} \alpha$ is the only line that is 


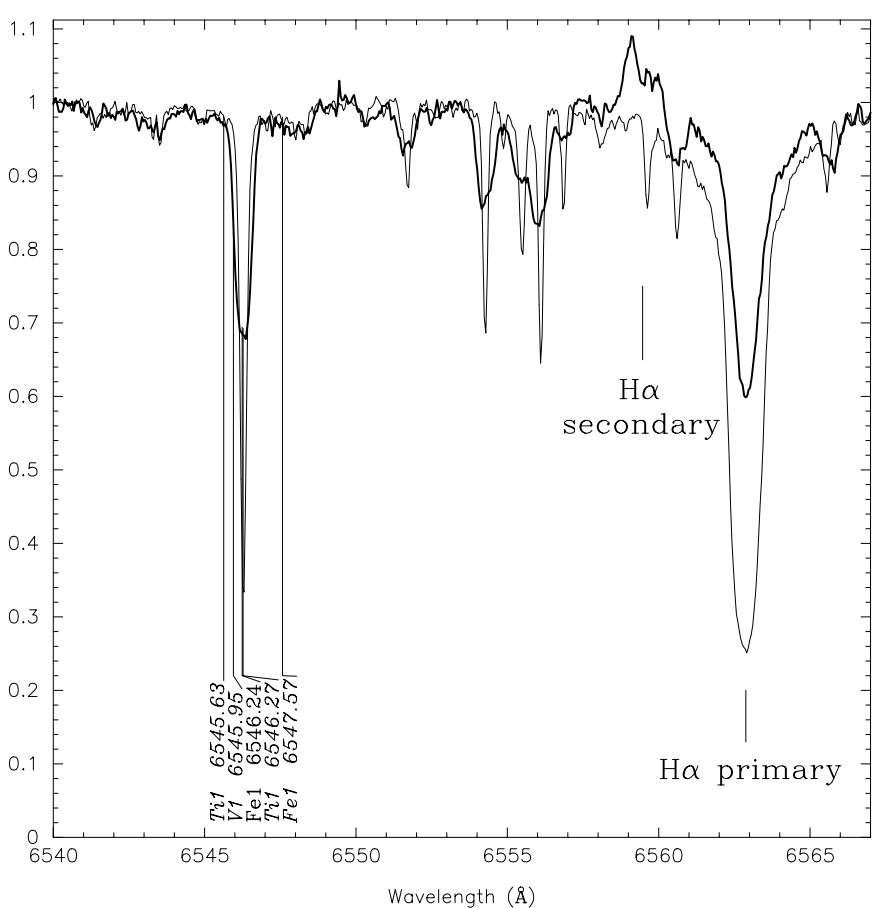

Fig. 1. A typical spectrum of AG Dor (thick line). The thin line shows the K0 V standard star HR 857. Note the blue-shifted $\mathrm{H} \alpha$-emission line from the secondary component and the filling of the primary-star line due to chromospheric emission. Also shown is the spectral line used for Doppler imaging the primary component at $6546.24 \AA$ along with four blends

detected from the secondary star and the accuracy of the secondary measurements is comparably poor. Its standard error of an observation of unit weight is $8.6 \mathrm{~km} \mathrm{~s}^{-1}$.

Our revised elements agree very well with the preliminary orbit (from L. A. Balona 1987; in Strassmeier et al. 1993). Our best orbits with minimized O-C residuals were adopted as the final solution, are given in Table 3 and plotted in Figs. 2 and 3 for UX For and AG Dor, respectively.

Phases of all line profiles were computed using revised ephemerises. For UX For

$\mathrm{HJD}=2448945.2348+0.954812 \times E$,

and for AG Dor

$\mathrm{HJD}=2448945.496+2.5620 \times E$.

The uncertainties of the zero point and the periods are such that we achieve a phase accuracy of 0?001 for UX For and 0 p.003 for AG Dor.

\subsection{Binary-spectrum synthesis}

Because the spectra of close binaries such as UX For and AG Dor appear as composites, we need to separate the two stellar spectra at all phases in order to extract activity features from each spectrum. This is done by generating an artificial composite spectrum from two non-active MK standard stars closely matching our object stars. A modified version of the computer program originally

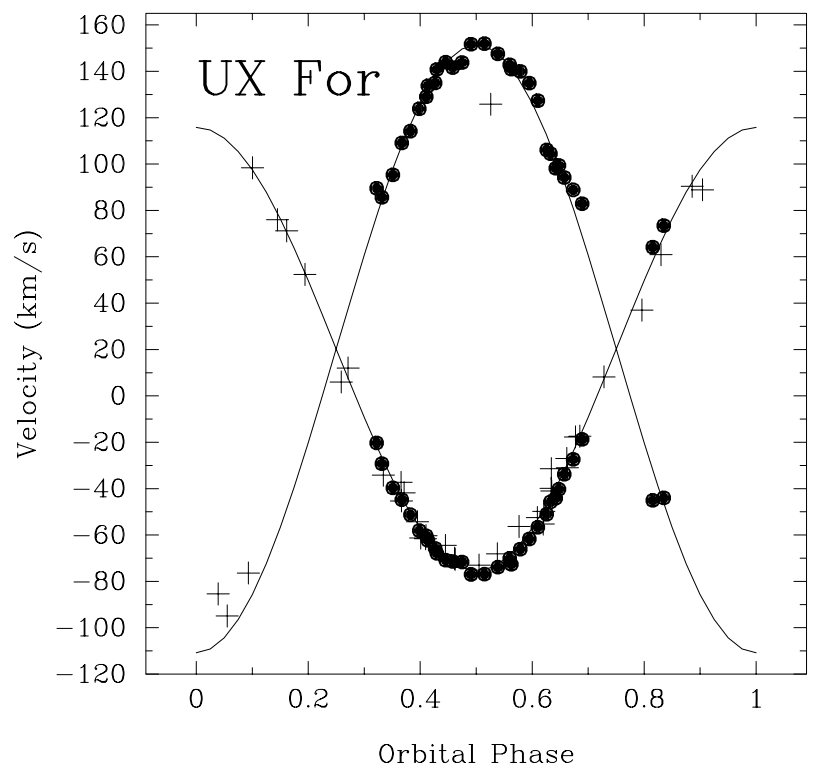

Fig. 2. Observed and computed radial velocity curves for UX For. Dots are the measurements from this paper, crosses are from Balona (1987)

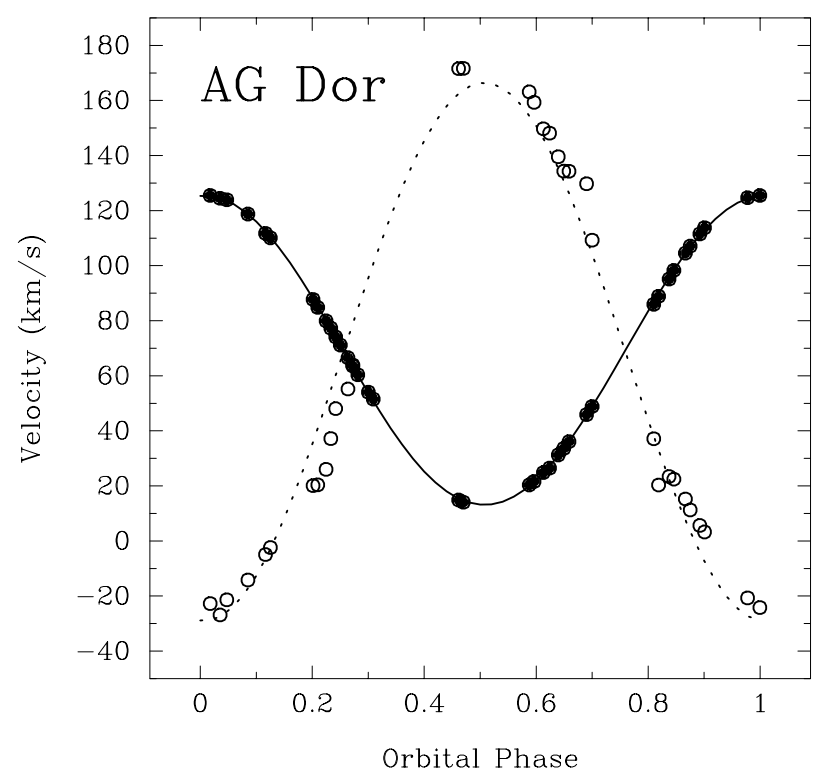

Fig. 3. Observed and computed radial velocity curves for AG Dor. Filled circles are the cross-correlation measurements of the primary star, open circles are the values of the residual $\mathrm{H} \alpha$ line of the secondary star

developed by Huenemoerder \& Barden (1984) (see also Huenemoerder et al. 1989) was used. The procedure includes rotational broadening, radial-velocity shifting, and intensity weighting of both standard-star spectra in the Fourier domain. The resulting difference spectrum is minimized by a least-squares approach. The input set of reference spectra then gives the overall best-fit combination (following Strassmeier \& Fekel 1990). Finally, we subtract the "synthetic" binary spectrum from each observed spectrum to eliminate the contribution from the underlying inactive part of the stellar photospheres and chromospheres. 
Table 3. Double-lined orbital elements for UX For and AG Dor. The preliminary values were taken from Balona (1987). Note that for UX For the elements were solved for both companions simultaneously while for AG Dor the primary elements were solved separately from the secondary elements

\begin{tabular}{|c|c|c|c|c|c|c|c|}
\hline \multirow[b]{2}{*}{ Element } & \multicolumn{3}{|c|}{ UX Fornacis } & \multicolumn{3}{|c|}{ AG Doradus } & \multirow[b]{2}{*}{ Unit } \\
\hline & Prelim. & Final & Error & Prelim. & Final & Error & \\
\hline$P_{\text {orb }}$ & 0.95479 & 0.9548120 & $510^{-7}$ & 2.562 & 2.5620 & 0.0027 & days \\
\hline$\gamma$ & 19.1 & 20.3 & 0.7 & 70.2 & 68.95 & 0.08 & $\mathrm{kms}^{-1}$ \\
\hline$K_{1}$ & 91.8 & 95.6 & 1.2 & 58.0 & 56.13 & 0.13 & $\mathrm{~km} \mathrm{~s}^{-1}$ \\
\hline$K_{2}$ & - & 131.0 & 2.8 & - & 97.9 & 1.6 & $\mathrm{~km} \mathrm{~s}^{-1}$ \\
\hline$e$ & 0 & 0 & - & 0.04 & 0 & - & \\
\hline$T_{0}$ & 4000.512 & 8945.2348 & 0.002 & 4378.67 & 8945.496 & 0.002 & $244+\mathrm{HJD}$ \\
\hline$f(M)$ & 0.077 & 0.087 & 0.003 & 0.052 & 0.0470 & 0.0003 & $M_{\odot}$ \\
\hline$M_{1} / M_{2}$ & - & 1.371 & 0.024 & - & 1.745 & 0.032 & \\
\hline$M_{1} \sin ^{3} i$ & - & 0.667 & 0.023 & - & 0.617 & 0.024 & $M_{\odot}$ \\
\hline$M_{2} \sin ^{3} i$ & - & 0.487 & 0.014 & - & 0.354 & 0.009 & $M_{\odot}$ \\
\hline$a_{1} \sin i$ & 1.21 & 1.255 & 0.015 & 2.04 & 1.977 & 0.005 & $10^{6} \mathrm{~km}$ \\
\hline$a_{2} \sin i$ & 1.48 & 1.721 & 0.037 & - & 3.45 & 0.06 & $10^{6} \mathrm{~km}$ \\
\hline $\begin{array}{c}\text { Standard error of an } \\
\text { observation of unit weight }\end{array}$ & & & 4.62 & & & $0.41 / 8.6$ & $\mathrm{~km} \mathrm{~s}^{-1}$ \\
\hline
\end{tabular}

Observed spectra of UX For and AG Dor, together with the respective synthesized spectra, were already shown in Washuettl \& Strassmeier (1995) and are not repeated here. In Fig. 4, we show the sequence of the 14 reference standard stars that are used for the synthesis, sorted by spectral type. Their classifications from the literature were uncertain and inhomogeneous and were partially revised in the course of this work. The criteria applied were the same as described in Strassmeier \& Fekel (1990), and are based on a number of temperaturesensitive line ratios and the morphology of the $\mathrm{H} \alpha$ profile. The adopted spectral types are indicated in Fig. 4.

\subsection{Spectral type, $v \sin i$, and relative intensity}

UX For. Among the 14 standard stars in Fig. 4, the combination $\alpha$ Men $(\mathrm{G} 6 \mathrm{~V})+o^{2}$ Eri $(\mathrm{K} 0.5 \mathrm{~V})$ fits the spectrum of UX For best, as judged from the overall goodness of fit $\left(\chi^{2}\right)$. A spectral type of about G5 for the combined spectrum was already favored by Houk (1982) and Bidelman \& MacConnell (1973), while Cutispoto (1998) opted for a combination of $\mathrm{G} 6 \mathrm{~V}+\mathrm{K} 3 \mathrm{~V}$. We also obtain a value for $v \sin i$ of the secondary star, $40 \pm 10 \mathrm{~km} \mathrm{~s}^{-1}$ (see Table 4), from line widths.

$A G$ Dor. We achieved best results with HR 857 (K0 V) and TW PsA (K4V) and an intensity relation of 1:19. AG Dor's spectra do not contain information about the photospheric secondary spectrum except its continuum contribution and $\mathrm{H} \alpha$-equivalent widths, and the continuum intensity weight is likewise uncertain. Cutispoto (1992, 1998, and private communication) suggests a spectral combination of $\mathrm{K} 1-2+\mathrm{K} 6, \mathrm{~K} 1+\mathrm{K} 5$, or even $\mathrm{K} 3+\mathrm{M}$ from photometric observations. $\mathrm{K} 0 \mathrm{~V}+\mathrm{K} 4 \mathrm{~V}$ implies a stellar radius ratio of $\approx 0.85$, according to Schmidt-Kaler (1982). Assuming that the two components corotate, this ratio also matches the relation of the $v \sin i$ values,
CALIBRATION STARS

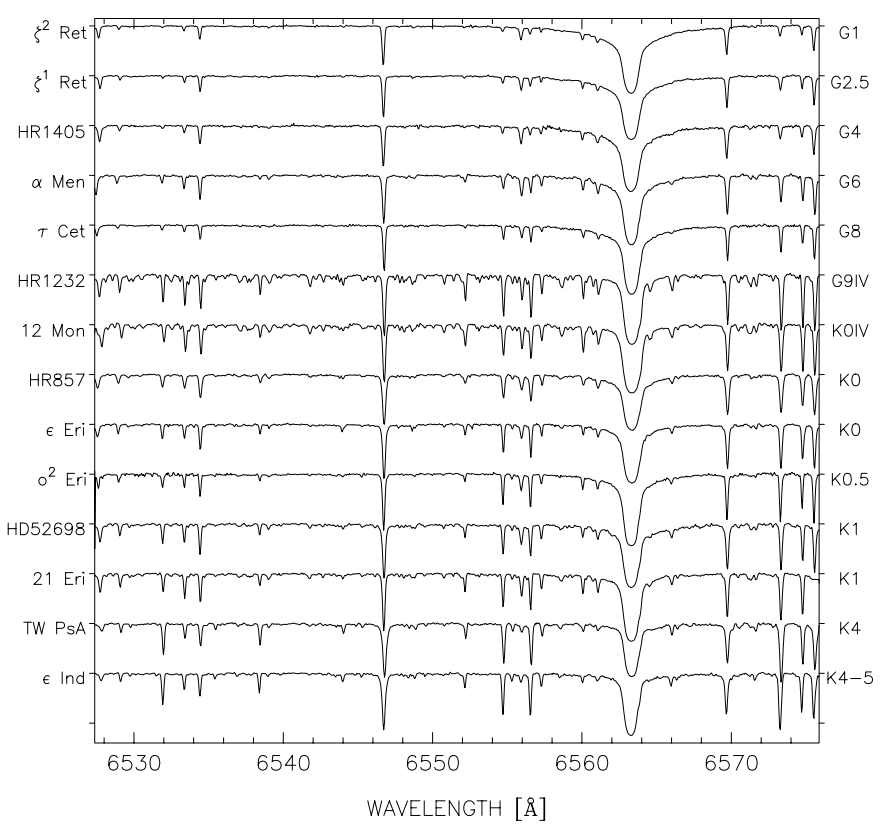

Fig. 4. The reference spectra used in the binary-star synthesis. Our improved classifications are listed on the right side of the plot. Note that the two spectra for the MKV standards HR 1232 and 12 Mon turned out to be subgiants instead of dwarfs

resulting in an upper limit for the secondary's $v \sin i$ of $14 \mathrm{~km} \mathrm{~s}^{-1}$. The spectrum-synthesis technique gives best results (i.e. smallest $\chi^{2}$ ) when a value of $10 \mathrm{~km} \mathrm{~s}^{-1}$ is chosen, in agreement with the expected upper limit. However, a $v \sin i$ of $10 \pm 5 \mathrm{~km} \mathrm{~s}^{-1}$ for the secondary implies a spectral type from mid-K to mid-M and reflects the uncertainty due to its extremely weak photospheric contribution. From estimates based on colour indices and the spectrum synthesis, we expect a spectral type of late $\mathrm{K}$ or 
Table 4. Summary of best-fit parameters from spectrum synthesis

\begin{tabular}{ccccc}
\hline & \multicolumn{2}{c}{ UX For } & \multicolumn{2}{c}{ AG Dor } \\
& Prim. & Sec. & Prim. & Sec. \\
\hline reference star & $\alpha$ Men & $\mathrm{o}^{2}$ Eri & HR 857 & TW PsA \\
spectral type & G6 V & K0.5 V & K0 V & K4 V \\
$v \sin i\left[\mathrm{~km} \mathrm{~s}^{-1}\right]$ & $48 \pm 2$ & $40 \pm 10$ & $17^{a} \pm 2$ & $10 \pm 5$ \\
$I_{\text {rel }}$ & 0.825 & 0.175 & 0.949 & 0.051 \\
\hline
\end{tabular}

a Preliminary value. See Sect. 5.1.

Table 5. Luminosities and radii based on the Hipparcos parallax (see Sect. 3.5) and $T_{\text {eff }}$

\begin{tabular}{lrrrr}
\hline & \multicolumn{2}{c}{ UX For } & \multicolumn{2}{c}{ AG Dor } \\
& Prim. & Sec. & Prim. & Sec. \\
\hline$m_{V}[\mathrm{mag}]$ & 8.16 & 9.84 & 8.61 & 11.78 \\
$M_{V}[\mathrm{mag}]$ & 5.13 & 6.81 & 5.89 & 9.07 \\
& \pm 0.07 & \pm 0.07 & \pm 0.06 & \pm 0.06 \\
$M_{\text {bol }}[\mathrm{mag}]$ & 4.99 & 6.21 & 5.54 & 7.94 \\
& \pm 0.07 & \pm 0.07 & \pm 0.06 & \pm 0.06 \\
$L\left[L_{\odot}\right]$ & 0.78 & 0.25 & 0.47 & 0.051 \\
& \pm 0.05 & \pm 0.01 & \pm 0.02 & \pm 0.001 \\
$T[\mathrm{~K}]$ & 5500 & $\approx 4500$ & 4900 & $\leq 4000$ \\
& \pm 200 & \pm 300 & \pm 100 & \\
$R\left[R_{\odot}\right]$ & 0.98 & 0.83 & 0.95 & $\geq 0.47$ \\
& \pm 0.08 & \pm 0.11 & \pm 0.05 & \\
\hline
\end{tabular}

M0, which is more consistent with a $v \sin i$ closer to the upper limit of $14 \mathrm{~km} \mathrm{~s}^{-1}$.

Table 4 summarizes the best-fit spectral types and parameters from the spectrum synthesis for both binaries.

\subsection{Effective temperatures from photometric colours}

We estimate the combined effective temperature of AG Dor by comparing observed $U-B$ and $B-V$ colours (Cutispoto 1998) to synthetic values from ATLAS-9 model atmospheres (Kurucz 1993) ${ }^{1}$. AG Dor's $U-B$ of 0.63 and $B-V$ of 0.91 suggest a $T_{\text {eff }}$ of $4750 \mathrm{~K}$, while the $V-R_{\mathrm{C}}$ of 0.55 leads to $T_{\text {eff }}=4830 \mathrm{~K}$. The $B-V$ vs. $T_{\text {eff }}$ calibration from Flower (1996) suggests $5025 \mathrm{~K}$. The effective temperatures can also be determined from the spectral types listed in Table 4 but are more uncertain and therefore not used. While the above temperatures are for the combined AG Dor binary system, they are dominated by the primary star (its spectrum provides at least $95 \%$ of the total light). Because the secondary star is cooler than the primary, the true temperature for the primary must be slightly higher than the combined temperature from both stellar components. We adopt a final value of $4900 \mathrm{~K}$ for the primary of AG Dor, which is in agreement with the results from Doppler imaging (Sect. 5). No lines of the secondary component are visible in our red wavelength

\footnotetext{
1 ftp://ccp7.dur.ac.uk/ccp7/Atlas/colours/ubvm05k2.dat
}

Table 6. Estimated radii and spectral types based on $P_{\text {rot }}$, $v \sin i$, and the empirical list of dwarf-star radii from SchmidtKaler (1982)

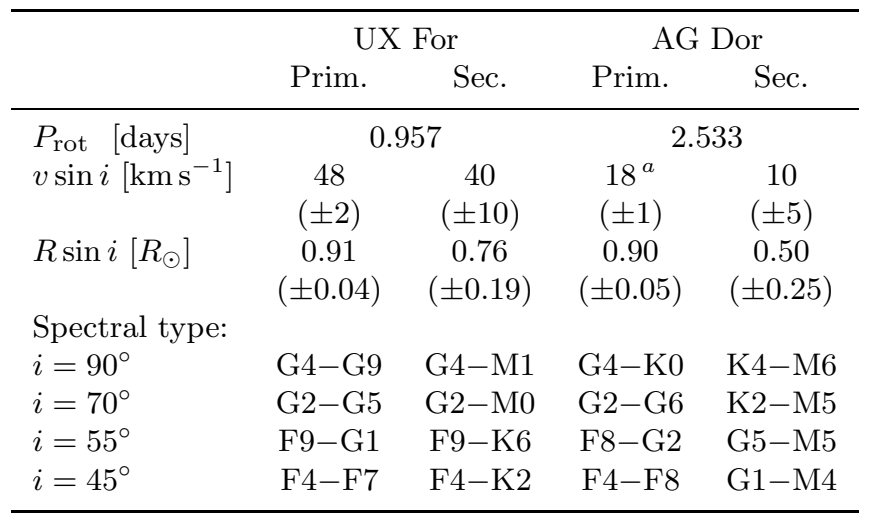

${ }^{a}$ From the Doppler imaging analysis in Sect. 5.1.

spectra. Therefore, we estimate a temperature of no more than $\approx 4000 \mathrm{~K}$ for the secondary.

For the combined system of UX For $(B-V=0.75$, Cutispoto 1998), Flower (1996) gives a temperature of $5413 \mathrm{~K}$, Schmidt-Kaler (1982) lists 5570 K, and Kurucz (1993) gives $5470 \mathrm{~K}$. Assuming a spectral type of G6 V for the primary, we obtain $T_{\text {eff }}=5500 \mathrm{~K}$ (contributing $82 \%$ of the light) and $\approx 4500 \mathrm{~K}$ for the secondary.

\subsection{Comparison with Hipparcos-based absolute parameters}

The Hipparcos spacecraft measured a trigonometric parallax of $28.67 \pm 0.83$ milli-arcsec for AG Dor and $24.77 \pm$ 0.80 milli-arcsec for UX For (ESA 1997), corresponding to distances of $34.9 \pm 1.0 \mathrm{pc}$ and $40.4 \pm 1.3 \mathrm{pc}$, respectively. The brightest (combined) $V$ magnitude of AG Dor was reported by Cutispoto (1998) to be 8.55 . UX For's brightest $V$ magnitude is 7.95 (see the CABS catalog; Strassmeier et al. 1993).

Using the normalized intensity relations obtained with spectrum synthesis (0.949:0.051 for AG Dor and 0.825:0.175 for UX For) we can calculate luminosities and radii for each component (Table 5). The visual magnitudes are $8^{\mathrm{m}} \cdot 61$ and $11^{\mathrm{m}} 78$ (AG Dor, primary and secondary) and 8.16 and 9.84 (UX For, primary and secondary). Combined with the distance, this gives the absolute visual magnitudes. The intensity errors were included in the absolute magnitudes, so the errors quoted merely reflect the error of the parallaxes. Adding the bolometric corrections from Flower (1996), -0.352 and -1.122 (primary and secondary) for AG Dor and -0.139 and -0.601 for UX For (neglecting interstellar absorption because they are nearby stars) leads to the bolometric magnitudes. Adopting a solar bolometric magnitude of 4.72 , we get the luminosities. With the temperatures from Sect. 3.4 and above luminosities, we obtain the stellar radii from the Stefan-Boltzmann law (independent of the inclination) and find $0.95 \pm 0.05 R_{\odot}$ and $\geq 0.47 R_{\odot}$ (AG Dor), in fair 
agreement with our previous estimations for $i=70^{\circ}$, and $0.98 \pm 0.08 R_{\odot}$ and $0.83 \pm 0.11 R_{\odot}(\mathrm{UX}$ For $)$

From the tables listed in Schmidt-Kaler (1982), this result is what we would expect for AG Dor from a combination of $\approx \mathrm{G} 4(\mathrm{G} 3-\mathrm{G} 6)+\mathrm{M} 2$ (or earlier), and for UX For from $\approx \mathrm{G} 3(\mathrm{G} 1-\mathrm{G} 6)+\mathrm{K} 1(\mathrm{G} 4-\mathrm{K} 5)$. For UX For these results are consistent within the error bars with the spectral types used for spectrum synthesis. For AG Dor, spectrum synthesis expects a later spectral type of $\mathrm{K} 0$ for the primary and mid-K to mid-M for the secondary (see Sect. 3.3), while the above estimation using stellar radii yields G3-G6 for the primary and - in good agreement an upper limit of M2 for the secondary. (See the discussion section.)

\subsection{Inclination}

We now can use the above radii, in combination with the semi-major axes from Table 3, to calculate the minimum values for the inclination angles for which no eclipses are observed $\left(R_{1}+R_{2}<a \cos i\right)$. We obtain $i<80^{\circ}$ for AG Dor and $i<67^{\circ}$ for UX For, in good agreement with Cutispoto (1998).

From $P_{\text {rot }}$ and $v \sin i$ we get $R \sin i$. Table 6 summarizes the results. Comparing these with the radii obtained from the Stefan Boltzman law in Sect. 3.5 (Table 5) we achieve an inclination of $71_{-8}^{\circ+19}$ for AG Dor and $68^{\circ+7}$ for UX For.

\section{4. $\mathrm{H} \alpha$ line variations}

\subsection{Residual $\mathrm{H} \alpha$-emission}

Residual H $\alpha$ spectra of UX For and AG Dor are generated by the subtraction of a synthesized inactive binary-star spectra. The resulting spectra can be considered as the chromospheric spectra of the object stars, and are shown as phase images in the top row of Fig. 5. The emission from both stellar components in both binary systems is seen as bright sinusoidal stripes (the horizontal grey zones mark holes in the phase coverage). The phase image of AG Dor also shows weak dark sinusoidal lines at the wavelengths of nearby absorption lines. This is due to an incomplete reproduction of the depths of some photospheric lines and could be due to a relative abundance mismatch between the object and the reference spectra.

Further detail is investigated in two ways, firstly, by measuring equivalent widths $(E W)$ of the residual $\mathrm{H} \alpha$ emission lines and, secondly, by subtracting the one residual spectrum with the least emission- $E W$ (we call these the normalized residual spectra). The remaining emission, if any, must be due to non-stationary features in the stellar chromosphere, like plages, flares, bright points and others.

\subsection{UX For}

Double-Gaussian fits to the residual spectra with IRAF's splot routine yield average $E W$ values of $588 \pm 73 \mathrm{~m} \AA$ for
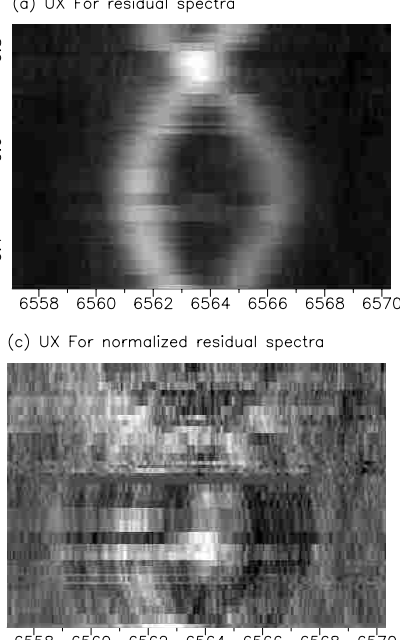

Fig. 5. Spectral phase images from the residual spectra of a) UX For and b) AG Dor. The $\mathrm{H} \alpha$ emission from both stellar components is seen as two sinusoidal stripes. For both binary systems the emission of the primary is stronger than that of the secondary. The normalized residual phase images are shown in c) for UX For and in d) for AG Dor, after subtracting the one residual spectrum with the smallest emission equivalent width. This should leave the non-stationary part of the chromospheric emission as the remainder

the primary and $407 \pm 42 \mathrm{~m} \AA$ for the secondary. The range of individual values is between $498-784$ and $250-477 \mathrm{~m} \AA$ for the primary and secondary, respectively. The scatter of a single measurement is $\sim 20 \%$. Due to severe blending of the residual emission profiles, the $E W$ values around phase 0.25 and 0.75 are not reliable and were not included in the calculation of above values.

To build the normalized residual spectra, we subtract the residual spectra with the spectrum from phase 0?6101. In this spectrum the two $\mathrm{H} \alpha$ emission peaks are the weakest within our data set, thus presumably representing the least-active phase, and they are well separated. Figure $5 \mathrm{c}$ plots the normalized spectra versus phase. Variations are seen in the stronger-emission component, e.g. between phase $\approx 0 \mathrm{p} 4$ and $0 \mathrm{p} 6$. These variations may be due to additional emission from plages or unresolved flares. There is also some evidence for additional emission between the two $\mathrm{H} \alpha$ components (the zero-velocity center) around phase 0.5 . This emission might be enhanced by errors in setting the continuum, as it is poorly defined between the primary and secondary $\mathrm{H} \alpha$-feature. However, tests with various continuum settings showed that it could not account for the total residual emission around 0 p.5. At that phase the intensity of the residual emission exceeds the signal-to-noise level at least by a factor of five. We suspect that it is caused by neutral hydrogen left over from coronal-mass ejections and "stored" near the inner Lagrangian point. A similar scenario was suggested for other chromospherically active binaries, e.g. for V471 Tau (Young et al. 1991).

The presence of circumstellar material or mass flow due to prominences or flares may dilute (or even supress) the 

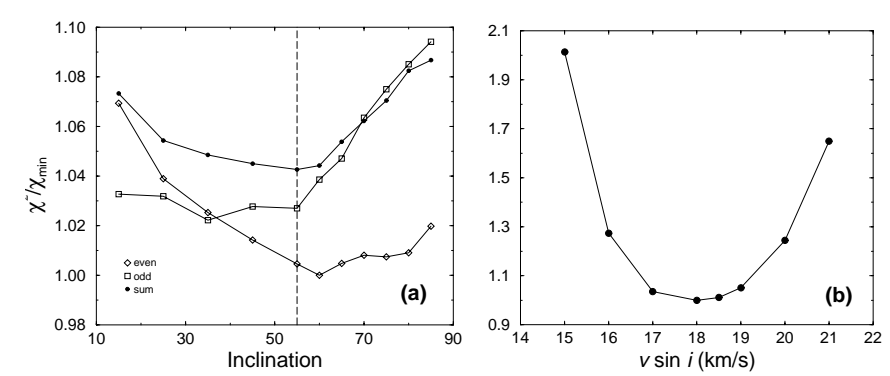

Fig. 6. The normalized goodness of fit for AG Dor (from Doppler imaging) as a function of a) the stellar inclination and $\mathbf{b}$ ) the projected rotational velocity. The minima indicate the chosen "best-fit" values

rotationally modulated signature. For both components of UX For, no clear phase dependency of the $\mathrm{H} \alpha$-variations could be found, but due to the limited phase coverage and signal-to-noise ratio of our spectra we can not fully exclude that $\mathrm{H} \alpha$ is rotationally modulated. On the Sun, we see that eruptive prominences change their shape on timescales of hours or even minutes, with radial velocities of several hundred $\mathrm{km} \mathrm{s}^{-1}$ (e.g. Tandberg-Hanssen 1995). If the $\mathrm{H} \alpha$-variations observed on UX For are due to eruptive prominences or flares, then it is likely that the relatively long exposure times of $20 \mathrm{~min}$ or more cannot resolve this activity.

\section{3. $A G$ Dor}

AG Dor's residual $\mathrm{H} \alpha$ emission shows only small or no variations. $E W$ values were measured by fitting a double Gaussian to the residual emission lines. These $E W$ values are only reliable when the separation of the emission lines is large enough so that no severe blending occurs. The average $E W$ for AG Dor is $428 \pm 27 \mathrm{~m} \AA$ and $168 \pm 32 \mathrm{~m} \AA$ for the primary and secondary, respectively, and the range of individual values is $387-511$ and $121-241 \mathrm{~m} \AA$ for the primary and secondary. The error per single measurement remains within $20 \%$. Spectral phases at $0.25 \pm 0.07$ and $0.75 \pm 0.07$ were excluded.

The spectra, after subtraction of the residual spectrum at phase 0 p.0178, are shown in Fig. 5d. As in the case of the $E W$ method, we found no rotational modulation of the $\mathrm{H} \alpha$ emission. The only noticeable variation is a transient absorption feature at phase $0.2-0$ ? 3 (observed in the same night). It passes through the combined $\mathrm{H} \alpha$-line from shorter to longer wavelengths, i.e. along the orbit of the secondary, but at a steeper angle, i.e. a higher velocity. We suspect that it might be caused by a circum-stellar cloud around the secondary, e.g. a corotating prominence, but because it occured only once and exactly at a time of inferior conjunction (where the degree of blending is most severe), we can not claim that it is a real detection.

\section{Doppler imaging of AG Doradus}

\subsection{Input parameters and assumptions}

The technique of Doppler imaging (see e.g. Rice 1996) offers the potential to resolve the spot distribution on the surfaces of rapidly-rotating stars. Many of the stars observed with this technique reveal spots in high latitudes, contradicting the solar paradigm. However, all of these stars have substantially larger rotation rates than the Sun because rotational broadening is required. The slowest rotator that has ever been successfully Doppler imaged is the G1.5 solar-type field star EK Dra (Strassmeier \& Rice 1998) with a $v \sin i$ of $17.3 \mathrm{~km} \mathrm{~s}^{-1}$ (but a period of 2.6 days). AG Dor has an almost identical projected rotational velocity as EK Dra, and is so far the slowest-rotating $\mathrm{K}$-dwarf that is being Doppler imaged.

The Doppler maps in this paper were generated using the temperature mapping code TempMap (Rice et al. 1989). The updated version was presented and tested by Rice \& Strassmeier (2000). We adopted a maximumentropy regularization for all inversions. A grid of ten model atmospheres with temperatures between 3500 and $5500 \mathrm{~K}$ in steps of $250 \mathrm{~K}$ and $\log g=4.5$ were taken from the ATLAS-9 CDs (Kurucz 1993). Gray (1992) lists a $\log g$ of 4.55 for a K1 dwarf. Trial inversions with other $\log g$ values did not result in better line-profile fits, thus $\log g=4.5$ was adopted. We can make use of only one (moderately) unblended line: Fe I $6546 \AA$ with a logarithmic transition probability $(\log g f)$ of -1.35 and a lower excitation potential of $2.76 \mathrm{eV}$. For the local line-profile tabulation, we synthesize a total of five blends (including the main mapping line) as indicated in Fig. 1. The secondary star contributes about $5 \%$ to the continuum intensity which decreases the apparent line depth of the primary star. Therefore, all spectra were multiplied by a factor of 1.05 in order to correct the contribution of the secondary star.

Figure 6 a shows the normalized $\chi^{2}$ distribution (normalized by the minimum value) from a series of test reconstructions as a function of the stellar inclination angle. We find an average minimum at around $i \approx 55^{\circ} \pm 10^{\circ}$ from the odd, even, and full data set (see next section). Kürster (1993), Unruh \& Collier Cameron (1995) and most recently Rice \& Strassmeier (2000), have shown that the minimization of $\chi^{2}$ can be successfully used to constrain the inclination angle. Of course, whether the best attainable fit to the data is also the correct one is debatable. We also note that the surface spot distribution nevertheless remains similar, even for a change of the stellar inclination of about $\pm 20^{\circ}$ (Vogt et al. 1987).

Figure $6 \mathrm{~b}$ shows the normalized $\chi^{2}$ distribution as a function of the projected rotational velocity. We find a clear minimum at $v \sin i=18 \pm 1 \mathrm{~km} \mathrm{~s}^{-1}$. The best $v \sin i$ with the spectrum-synthesis method was $17 \pm 2 \mathrm{~km} \mathrm{~s}^{-1}$, while Pallavicini et al. (1992) obtained $20 \mathrm{~km} \mathrm{~s}^{-1}$. However, the Doppler-imaging technique is very sensitive to the projected rotational velocity and provides better accuracy than any other method, as it takes 

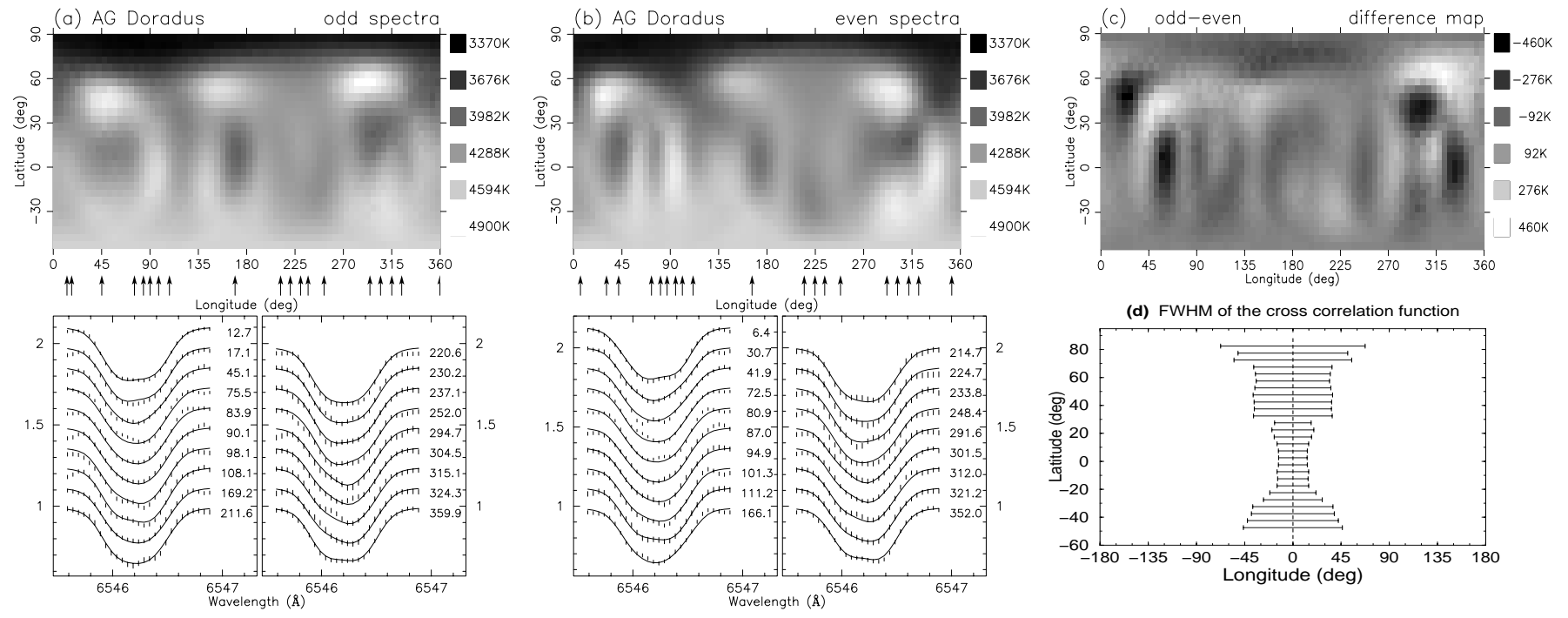

Fig. 7. Doppler images in pseudo-mercator projection for the Fe I $6546.239 \AA$ line. a) odd spectra, b) even spectra. The arrows below the temperature maps indicate the respective phase coverage. The subpanels show the observed and computed line profiles. The lines are the fits and the bars are the observations. The length of a bar indicates the $S / N$ per pixel. c) is the difference-map from the odd and even images. d) shows the $F W H M$ of the cross correlation function from the odd and even map per latitude bin

Table 7. Adopted Doppler-imaging parameters for AG Dor

\begin{tabular}{ll}
\hline Parameter & Value \\
\hline$T_{\text {phot }}$ & $4900 \mathrm{~K}$ \\
$\log g$ & 4.5 \\
$v \sin i$ & $18 \mathrm{~km} \mathrm{~s}^{-1}$ \\
$i$ & $55^{\circ}$ \\
$P_{\text {rot }} \equiv P_{\text {orb }}$ & 2.562 days \\
Micro turbulence $\xi$ & $2.0 \mathrm{~km} \mathrm{~s}^{-1}$ \\
Macro turbulence $\zeta_{\mathrm{R}}=\zeta_{\mathrm{T}}$ & $4.0 \mathrm{~km} \mathrm{~s}^{-1}$ \\
{$[\mathrm{Fe} / \mathrm{Fe} \odot]$} & -0.45 \\
\hline
\end{tabular}

into account blends in the line profile as well as line deformation due to spots. We therefore adopt $18 \pm 1 \mathrm{~km} \mathrm{~s}^{-1}$ as the most likely $v \sin i$ value.

The average $S / N$ ratio of $\approx 150: 1$ is low but still sufficient for Doppler imaging. The resolving power of 50000 yields a velocity resolution of $6 \mathrm{~km} \mathrm{~s}^{-1}$, offering six spectral resolution elements across the full width of the lines at the continuum. Numerical tests have shown that reliable images can be reconstructed with as low as 5 resolution elements (Piskunov \& Wehlau 1990; Hatzes 1993; Strassmeier \& Rice 1998). The relatively long rotational period, combined with an average integration time of $35 \mathrm{~min}$, allows for a surface phase resolution of around $3.5^{\circ}$ near the stellar meridian and along the direction of rotation, approximately a factor of ten smaller than the spectroscopic resolving power. The smearing due to orbital motion amounts to $\approx 3 \mathrm{kms}^{-1}$ for a 35 min integration, which is about half the size of the spectral resolution. We can therefore safely neglect phase smearing due to stellar rotation and orbital revolution.

\subsection{A Doppler image for November 1992}

We obtained 38 spectra from 6 consecutive nights and split them into two blocks of 19 spectra each, taking odd numbers for the first block and even numbers for the second (in chronological order). Therefore, we obtain two Doppler images from two independent data sets - but from the same epoch - and compare them as a consistency check (see also e.g. Barnes et al. 1998). However, our final maps are computed from the combined data.

Figure 7 compares the maps from the odd and even spectra. It demonstrates that both data sets reconstruct a very similar spot configuration with much detail in common. A bridge between the low-latitude spot at $\ell \approx 300^{\circ}$ and the polar appendage at $\approx 350^{\circ}$ is visible in both cases (it is a bit more emphasized in the even map). Also an equatorial spot at $\ell \approx 180^{\circ}$ and an asymmetry of the polar spot at $\ell \approx 100^{\circ}$ is visible. A third low-latitude feature at $\ell \approx 50^{\circ}$ appeared split into two cool features in the even-spectra image, while single and of lower contrast in the odd-spectra image. The combined image still recovers a split feature with both parts of lower contrast compared to the even-spectra image. The difference between the odd and the even map in Fig. 7c shows an average divergence of $\pm 200 \mathrm{~K}$. This is mostly due to shifts of the spot location between the even and the odd map (above all from the bright patches, see below). Figure $7 d$ gives the $F W H M$ of the cross-correlation of the odd and even map as a function of stellar latitude. As expected, the equatorial latitudes show more details in the surface structure, resulting in a smaller $F W H M$ of the cross-correlation peak.

The observed and computed line profiles for the combined spectra, along with our final Doppler image, are plotted in Figs. 8a and 8b, respectively. We find a polar spot with two cool appendages having a temperature 

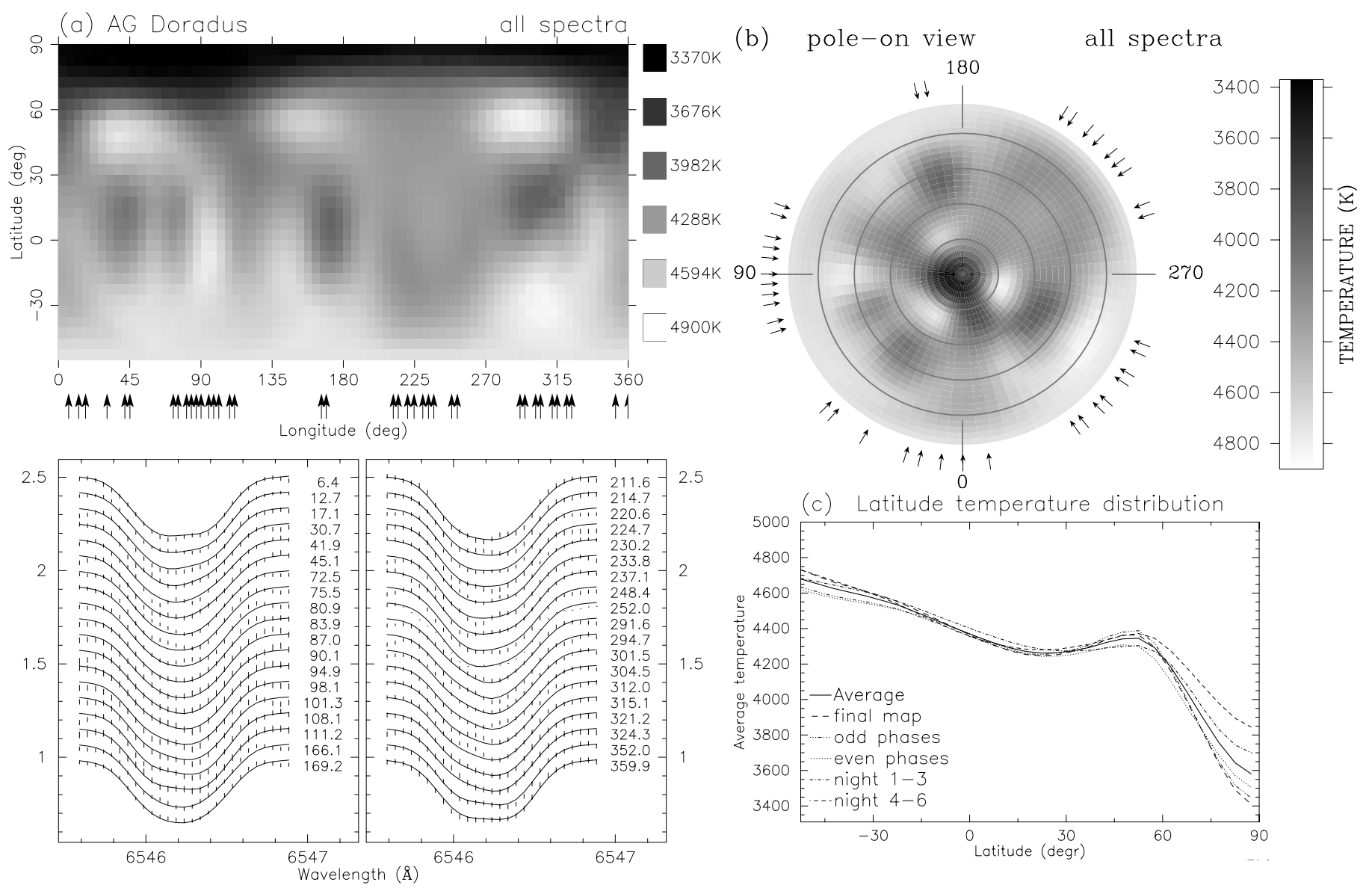

Fig. 8. Doppler images for Fe I 6546.239 ̊̊ line using all spectra a) in pseudo-mercator projection (the subpanel shows observed and computed line profiles) and b) in pole-on view. c) shows the average temperature distribution along constant latitudes. Arrows indicate the total phase coverage
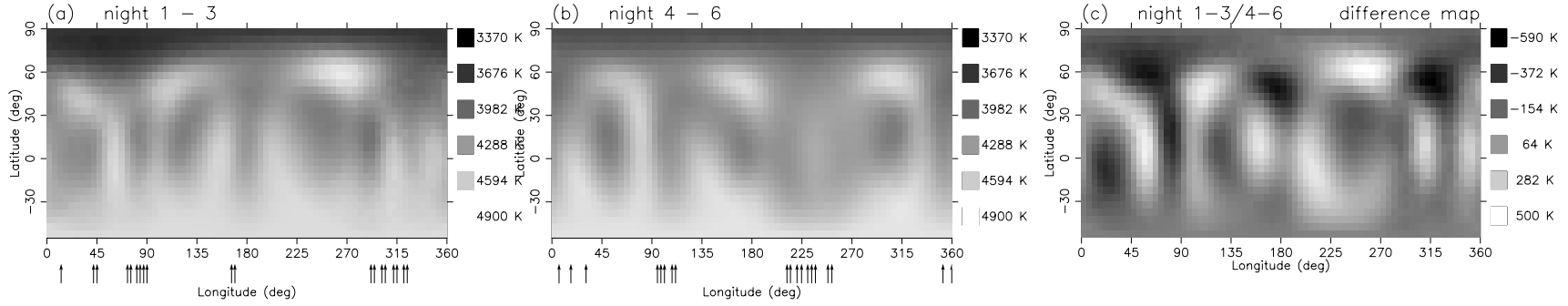

Fig. 9. Doppler image from a) the first and b) the second stellar rotation. c) shows the difference-map from a) and b)

difference of $1000-1500 \mathrm{~K}$ with respect to the adopted photospheric temperature of $4900 \mathrm{~K}$. A total of three low-latitude spots or spot groups are recovered with a temperature difference of $\approx 800 \mathrm{~K}$. Two or possibly three high-latitude bright patches $(T \approx 4860-4930 \mathrm{~K})$ seem to occur at the same Doppler shifts as the three cool equatorial spots. The difference map from the odd and even spectra in Fig. 7c shows the largest differences at the location of two of these bright patches, indicating that these features are very likely artificial due to the combined effects of small $v \sin i$, limited $S / N$, and a mirroring with the cool spots.

Figure 8c shows the latitudinal temperature distribution. It was derived by binning all surface pixels along constant latitudes. The average temperature in each bin is generally below the expected photospheric temperature of $4900 \mathrm{~K}$. A relatively sharp drop in temperature occurs at a latitude of approximately $50-60^{\circ}$, close to the projected angle of inclination. However, this is merely coincidental and due to the two polar appendages. The polar feature itself extends on average to a latitude of $+70^{\circ}$ (better seen in the pole-on projection in Fig. 8b), but it is clear that the high latitudes block most of the otherwise "missing flux".

The overall goodness of fit for the entire data set was $\chi_{\text {tot }}^{2}=0.2235$ (for the odd-spectra fit 0.1101 ; even-spectra fit 0.1077). These can be considered excellent fits for the $S / N$ ratio given. Some line profiles could not be fitted to the level we would have liked, notably phases $0 ! 116,0$ ? 210 , 0.846 and 0.875 in Fig. 8a. It is possible that the spot 
configuration slightly evolved within the six nights of our observations, i.e. two and a half stellar rotations. Therefore, we generated another set of two Doppler images, using the first 19 spectra (nights 1-3) and the second 19 spectra (nights $4-6$ ). The maps and the phase coverages are shown in Figs. 9a and b. Unfortunately, the phase gaps become very large (up to $120^{\circ}$ ), and it is not straightforward to compare the images in the presence of such large gaps (see Rice \& Strassmeier 2000). We can not draw any firm conclusions whether the differences are solely due to the poor reconstruction within the phase gaps or due to partial spot migrations on the stellar surface. Cross-correlating the two surface maps also did not lead to a useful correlation because of the masking with the phase gaps.

\section{Summary and discussion}

Both binary systems under investigation were found to have active secondaries. In UX For, the absorption spectrum of the secondary was detected and a regular doublelined spectroscopic orbit computed. In the case of AG Dor, we found no photospheric lines from the secondary, but detected its strong $\mathrm{H} \alpha$ emission and used it to determine a double-lined orbit. Both secondary stars are later in spectral type than their respective primaries, G6+K0.5 for UX For and $\mathrm{K} 0+\mathrm{K} 4$ for $\mathrm{AG}$ Dor, which makes them appear relatively more active due to their deeper convective envelopes. Both stars in both binary systems rotate synchronously to their respective orbital motion.

Our spectrum-disentangling technique as well as the Hipparcos parallax suggest that all components are on the main sequence or at least very close to it. Different approaches to determine absolute stellar parameters yielded radii, temperatures, and luminosities that are formally in agreement with each other, but yield a slightly inconsistent inclination for AG Dor with respect to our Doppler imaging analysis. Taking the quite reliable orbital elements (primary-lines velocity residual of $410 \mathrm{~ms}^{-1}$ ), we may estimate masses to within $4 \%$, mainly depending upon inclination. An inclination of $55^{\circ} \pm 10^{\circ}$ is suggested from Doppler imaging and would give $\approx \mathrm{dG} 0+\mathrm{dK} 5$ for AG Dor. On the other hand, an early $\mathrm{K}$ primary - expected from photometric colours - requires an inclination of between $65^{\circ}$ to $70^{\circ}$, thus being just marginally consistent with the inclination suggested from Doppler imaging. Geometrical constraints involving $P_{\text {rot }}, v \sin i$ and $i$ also favour an early or mid-G type main-sequence primary, while Doppler imaging, spectrum synthesis, broad-band colours and the Hipparcos distance emphasize a $\mathrm{K} 1 \pm 1$ primary and a K4 or K5 secondary. The discrepancy possibly indicates a slightly off-main-sequence evolutionary stage for the primary. The low lithium abundance of approximately half of the solar abundance likely excludes a pre-main-sequence status.

$\mathrm{H} \alpha$-line variations are detected in all four stars but none of them appeared periodic. The variations can be as large as a factor of two in equivalent width for the UX For primary but were below 30-40\% for the AG Dor primary. On the other hand, the secondary star of UX For appeared more constant than the secondary in AG Dor, a maximum of $50 \%$ variation compared to $100 \%$ for the AG Dor secondary, but their equivalent widths are also more uncertain than those of the primaries. The residual $\mathrm{H} \alpha$-line profiles of the two UX For components normalized to the spectrum with the weakest emission equivalent width show a transient emission during quadrature at the relative zero-velocity wavelength, i.e. near the inner Lagrangian point. Since the emission is only detected in three of our 40 spectra and is only observed at one particular epoch (1996.88), we can not draw any firm conclusions about it. However, if real, we suspect that it is caused by neutral hydrogen expelled from both active stars and stored in the inner Lagrangian point, where it is heated by the stellar coronal X-ray and UV emission.

Despite the fact that the two UX For components show more rapid rotation than AG Dor, our data allowed just the primary of AG Dor to be Doppler imaged. AG Dor shows a single-lined absorption spectrum and thus less blending. Its spectra have, at the same time, a $S / N$ ratio higher by a factor of two compared to UX For. Phase smearing due to the orbital motion during our relatively long integration times is also prohibitive for Doppler imaging of UX For, but no significant problem for AG Dor. However, mapping AG Dor still presents a challenge because only one photospheric line is available (Fe I $6546 \AA$ ) and the stellar rotational velocity of $v \sin i=18 \mathrm{~km} \mathrm{~s}^{-1}$ is at the limit of current mapping techniques, given the instrumental resolution of 50000 . Therefore, we included several tests to ensure that we minimized surface artifacts down to the level comparable with the intrinsic quality of the data.

A moderately sized polar spot along with four, possibly five, low-to-medium latitude spots are reconstructed from Fe I $6546 \AA$. The polar feature is quite asymmetric and has one, possibly two, appendages connecting it with lowerlatitude spots. This seems to be a commonly observed feature in spotted stars of various masses and ages (post and pre-main sequence). Amado et al. (1999) used multicolour broad-band photometry of AG Dor to reconstruct a spot map for winter 1996/97, and found a spot distribution very similar to ours. Modelling optical and infrared colour curves, they obtained effective temperatures as well as spot locations and sizes and found two low-latitude spots as well as a non-modulating component. They reconstructed it with a cool polar spot, which is also supported by their $\mathrm{TiO}$ molecular-band measurements. We may also note that the Doppler image of AG Dor appears quite similar to the Doppler image of the single, young, solar-type star EK Draconis (Strassmeier \& Rice 1998), as well as to the pre-main-sequence binary V824 Ara (Hatzes \& Kürster 1999; Strassmeier \& Rice 2000). It has been discussed whether polar features are connected to rapid rotation (e.g. Vogt et al. 1999). AG Dor and EK Dra are the two slowest rotators Doppler imaged so far (both are main-sequence stars and possibly not directly comparable to the active giants in $\mathrm{RS} \mathrm{CVn}$ binaries), and both of 
them show a polar feature whose center is displaced from the rotational axis along with several low-latitude or equatorial spots. This could be evidence that the surfacing latitudes of magnetic flux tubes coming from the bottom of the convection zone are being rapidly redistributed by surface velocity fields such as differential rotation, meridional flows, and sporadic plasma rearrangements triggered by magnetic reconnections. Several recent papers presented evidence for the existence of such surface velocity fields on spotted stars (see the review by Collier Cameron 2000; and also Weber \& Strassmeier 2000 for a most recent application). Binarity per se seems to play just a secondary role despite of these star's additional angular momentum due to spin-orbit coupling. For a discussion of the impact of tidal forces on the magnetic flux tubes in a close binary system, see Holzwarth \& Schüssler (2000).

Finally, we note that there seems to be no apparent correlation between the $\mathrm{H} \alpha$ variations on both binary components of AG Dor and our Doppler map of the primary.

Acknowledgements. We are very grateful to the Austrian Fonds zur Förderung der wissenschaftlichen Forschung for support under grant S7302-AST and S7301-AST and to the Deutsche Forschungsgemeinschaft for grant HU 532/8-1. We also thank M. Kürster (then MPE, now ESO) for his kind help with the remote CAT observations from Garching and G. Cutispoto for recalculating the colour indices of AG Dor. Special thanks go to Michael Weber and János Bartus for their personal support and computer assistance.

\section{References}

Amado, P. J., Butler, C. J., \& Byrne, P. B. 1999, MNRAS, 310,1023

Balona, L. A. 1987, S. Afr. Astr. Obs. Circ., 11, 1

Barker, E. S., Evans, D. S., \& Laing, J. D. 1967, ROB, No. 130

Barnes, J. R., Collier Cameron, A., Unruh, Y. C., Donati, J. F., \& Hussain, G. A. J. 1998, MNRAS, 299, 904

Bidelman, W. P., \& MacConnell, D. J. 1973, AJ, 78, 687

Blumberg, R. E., \& Boksenberg, A. (ed.) 2000, The Astronomical Almanac, H42

Bopp, B. W., Dempsey, R. C., \& Maniak, S. 1988, ApJS, 68, 803

Bowyer, S., Lampton, M., Lewis, J., et al. 1996, ApJS, 102, 129

Catalano, S., Rodonó, M., Frasca, A., \& Cutispoto, G. 1996, in IAU Symp. 176, Stellar Surface Structure, ed. K. G. Strassmeier, \& J. L. Linsky (Kluwer Acad. Press), 403

Collier Cameron, A. 2000, in Astrotomography, Lecture Notes in Physics, ed. H. Boffin \& D. Steeghs (Springer), in press

Collier Cameron, A., \& Robinson, R. D. 1989, MNRAS, 236, 57

Cutispoto, G. 1992, A\&AS, 95, 397

Cutispoto, G. 1998, A\&AS, 131, 321

Doyle, J. G., Butler, C. J., Byrne, P. B., et al. 1989, A\&A, 223, 219

ESA 1997, The Hipparcos and Tycho Catalogues, ESA SP-1200

Fekel, F. C., Strassmeier, K. G., Weber, M., Washuettl, A., \& Scheck, M. 1999, A\&AS, 137, 369

Fitzpatrick, M. J. 1993, in Astronomical Data Analysis Software and Systems II, ed. R. J. Hanish, R. V. J. Brissenden, \& J. Barnes, PASPC, 52, 472
Flower, P. J. 1996, ApJ, 469, 355

Gray, D. F. 1992, The Observation and Analysis of Stellar Photospheres (Cambridge: CUP), 430

Giampapa, M., Cram, L. E., \& Wild, W. J. 1989, ApJ, 345, 536

Hatzes, A. P. 1993, ApJ, 410, 777

Hatzes, A. P. 1998, A\&A, 330, 541

Hatzes, A. P., \& Kürster, M. 1999, A\&A, 346, 432

Henry, T. J., Soderblom, D. R., Donahue, R. A., \& Baliunas, S. L. 1996, AJ, 111, 439

Holzwarth, V., \& Schüssler, M. 2000, AN, 321, 175

Houk, N., Cowley, A. 1975, Michigan Catalogue of Two Dimensional Spectral Types for the HD Stars (Ann Arbor: Astr. Dept.), vol. 1

Houk, N. 1978, Michigan Catalogue of two-dimensional Spectral Types for the HD stars, Department of Astronomy, University of Michigan, Ann Arbor, vol. 2

Houk, N. 1982, Michigan Catalogue of two-dimensional Spectral Types for the HD stars, Department of Astronomy, University of Michigan, Ann Arbor, vol. 3

Huenemoerder, D. P., \& Barden, S. C. 1984, BAAS, 16, 510

Huenemoerder, D. P., Buzasi, D. L., \& Ramsey, L. W. 1989, AJ, 98, 1398

Kurucz, R. L. 1993, ATLAS-9, CD-ROM \# 13

Kürster, M. 1993, A\&A, 274, 851

Lloyd-Evans, T., \& Koen, M. C. J. 1987, S. Afr. Astr. Obs. Circ., 11, 21

Lucy, L. B., \& Sweeney, M. A. 1971, AJ, 76, 544

Oliveira, J. M., \& Foing, B. H. 1999, A\&A, 343, 213

Pallavicini, R., Randich, S., \& Giampapa, M. S. 1992, A\&A, 253, 185

Piskunov, N. E., \& Wehlau, W. H. 1990, A\&A, 233, 497

Pye, J. P., McGale, P. A., Allan, D. J., et al. 1995, MNRAS, 274,1165

Randich, S., Gratton, R., \& Pallavicini, R. 1993, A\&A, 273, 194

Rice, J. B. 1996, in IAU Symp. 176, Stellar Surface Structure, ed. K. G. Strassmeier, \& J. L. Linsky (Kluwer Acad. Press), 19

Rice, J. B., \& Strassmeier, K. G. 2000, A\&AS, 147, 151

Rice, J. B., Wehlau, W. H., \& Khokhlova, V. L. 1989, A\&A, 208, 179

Rodonò, M., Byrne, P. B., Neff, J. E., et al. 1987, A\&A, 176, 267

Schmidt-Kaler, Th. 1982, in Landolt-Börnstein, vol. 2b, ed. K. Schaifers, \& H. H. Voigt (Springer, Berlin), 31

Strassmeier, K. G. 1994, A\&A, 281, 395

Strassmeier, K. G., \& Fekel, F. C. 1990, A\&A, 230, 389

Strassmeier, K. G., Hall, D. S., Fekel, F. C., \& Scheck, M. 1993, A\&AS, 100, 173

Strassmeier, K. G., \& Rice, J. B. 1998, A\&A, 330, 685

Strassmeier, K. G., \& Rice, J. B. 2000, A\&A, 360, 1019

Strassmeier, K. G., Washuettl, A., \& Rice, J. B. 1994, IBVS, 3994

Tandberg-Hanssen, E. 1995, The Nature of Solar Prominences (Kluwer Acad. Publishers), 101

Unruh, Y. C., \& Collier Cameron, A. 1995, MNRAS, 273, 1

Vogt, S. S., Hatzes, A. H., Misch, A. A., \& Kürster, M. 1999, ApJS, 121, 547

Vogt, S. S., Penrod, G. D., \& Hatzes, A. H. 1987, ApJ, 321, 496

Washuettl, A., \& Strassmeier, K. G. 1995, in PosterProceedings IAU Symp. 176, Stellar Surface Structure, ed. K. G. Strassmeier, University of Vienna, 172

Weber, M., \& Strassmeier, K. G. 2001, A\&A, submitted

Young, A., Rottler, L., \& Skumanich, A. 1991, ApJ, 378, L25 